\title{
Topological Insulators and Superconductors and Mathematical Science
}

\section{by Shou-Cheng Zhang*}

\begin{abstract}
Topological insulator and superconductor are novel states of quantum matter whose low energy properties are described by topological field theory (TFT). Recently, TFT has directly led to the theoretical prediction of topological materials. In this contribution, I shall briefly review these recent developments and show that beautiful math inspires great discoveries in material science.
\end{abstract}

It is a distinct honor for me to be invited to speak at the opening of the Center for Mathematical Science at Harvard. Throughout history, crossfertilization among different branches of knowledge has often led to great advances in science. Mathematics played a central role in formulating the fundamental laws of physics. Topology seems to be a rather abstract branch of mathematics, but in recently years, it has found profound applications in material science. I am a condensed matter theorist working on the discovery of new states of matter in nature. In physics we take two distinct approaches to understand natural phenomena around us. One approach tries to break matter into smaller and smaller building blocks. More than one hundred years ago, discovery of the periodic table of elements led to the golden age of chemistry. In particle physics, accelerators with ever increasing energy probe smaller and smaller scales of the sub-atomic world. Another approach to science investigates the different states of matter which can

\footnotetext{
* Department of Physics, Stanford University, Stanford, Calif., U.S.A.

E-mail: sczhang@stanford.edu
}

be formed by the same building blocks. For example, the same $\mathrm{H}_{2} \mathrm{O}$ molecules can form three different phases of ice, water and steam. These forms of matter can only transfer into one another by a sharp phase transition. Electrons in solids interact with the atomic nuclei and with each other to form semi-conductors, magnets and superconductors. Some of these materials play important roles in information technology.

In mathematics one can classify geometric shapes by different symmetries. For example, Platonic solids are classified by the point group symmetries. Similarly, distinct states of matter can often be classified by the symmetries in the ordered and disordered phases. In the disordered state of a magnet where the magnetic moments point in random directions, the system is fully rotationally invariant. On the other hand, in the ordered phase, the magnetic moments line up to point in a particular direction, and the rotational symmetry is spontaneously broken. If the full symmetry group of the disordered state is $G$, and the remaining symmetry of the order phase is $H$, then the system is described by a field theory mapping the space-time into the coset manifold $G / H$. Majority of states encountered in condensed matter can be classified and described by this principle of spontaneous symmetry breaking.

The discovery of the quantum Hall effect has fundamentally changed the perspective of how condensed matter states are classified and described. A two dimensional electron gas can be realized at the interface between two semiconductors. At low temperature and with a high external magnetic field, the Hall conductance takes only quantized integer or 
fractional values in units of the fundamental constant $e^{2} / h$, where $e$ is the electron charge and $h$ is the Planck constant. It was realized that the quantized values are topological invariants, namely Chern numbers, associated with a $U(1)$ fiber bundle over the Brillouin zone torus of the electronic wave function. The low energy physics of the quantum Hall effect is described by a topological field theory (TFT), namely the ChernSimons theory [5].

For a while, the quantum Hall state seemed like a special case in nature where the topological classification and description are essential, while all other condensed matter systems are described by the principle of symmetry breaking. But the recently, new class of topological materials have been theoretically predicted and experimentally observed [1, 2, 3, 4]. Topological insulators are time reversal invariant systems where bulk states are separated by an energy gap but gapless surface states connect the bulk valence and conduction bands. These novel condensed matter systems can be generally described by TFT. Topological insulator materials are described by a topological term in the electromagnetic response [6] with an axion angle of $\theta=\pi$. In the case of the quantum Hall effect, TFT was introduced after the experimental discovery, however, in the recent developments, TFT played a vital role in predicting new topological materials $[7,8]$.

Chern-Simons theory was the first TFT introduced to physics $[9,10,11]$. Such a topological term appears dynamically if we couple the external electromagnetic field $A_{\mu}$ to the massive Dirac fermions in $2+1$ dimensions. Dirac fermions take two components in $2+1$ dimensions, and the mass term $M$ breaks time reversal symmetry. If we integrate out the Dirac fermion with some UV cut-off in the background of the external electromagnetic field, we obtain the Chern-Simons term [10, 11]:

$$
S_{\mathrm{eff}}=\frac{C_{1}}{4 \pi} \int d^{2} x d t \epsilon^{\mu \nu \tau} A_{\mu} \partial_{v} A_{\tau},
$$

with

$$
C_{1}=\frac{M}{2|M|}
$$

The current is defined by the variational derivative of $S_{\text {eff }}$ with respect of $A_{\mu}$, and is given by $J_{\mu}=\frac{C_{1}}{2 \pi} \epsilon^{\mu \nu \tau} \partial_{v} A_{\tau}$. This equation describes the Hall effect, and the electric current flows in a direction perpendicular to the applied electric field in two dimensions. This result is absolutely remarkable, equation (2) shows that something very strange happens when we change the sign of the Dirac mass. In fact, the result (2) depends on the UV cutoff, and is not universal-the value of $C_{1}$ can be anything depending on the cutoff. However, the jump of $C_{1}$

$$
\Delta C_{1}=1
$$

as one changes the sign of the mass is universal and independent of the UV cutoff. Restoring conventional units, $\Delta C_{1}=1$ implies a jump of the Hall conductance of $\Delta \sigma_{x y}=e^{2} / h$.

Both the integer and the fractional quantum Hall effect can be described by a low energy effective theory called the Chern-Simons-Landau-Ginzburg theory [5]. The topological response of the quantum Hall state can be entirely described by the TFT based on the Chern-Simons term (1). Electrons in lowest Landau level are very different from the massive Dirac fermion, we arrived at the TFT by first mapping electrons in the lowest Landau level into composite bosons by attaching an odd number of flux tubes. Such an operation is mathematically implemented by an internal gauge field described by the Chern-Simons term. Since the electrons became composite bosons, they can condense into a bose superfluid when the internal gauge field cancels the external electromagnetic field at the mean field level, which is the condition for the quantum Hall effect within our theory. Compared with the wave function description of Laughlin, the TFT can be much easily applied to non-trivial two dimensional manifold with higher genus. Based on the counting of ground state degeneracy of the TFT on manifolds with higher genus, Wen introduced the idea of topological order.

Fascinated by the power and simplicity of the TFT based on the Chern-Simons term, one can proceed formally, by considering the next Chern-Simons term, which exists in $4+1$ dimensions, given by

$$
S_{\text {eff }}=\frac{C_{2}}{24 \pi^{2}} \int d^{4} x d t \epsilon^{\mu \nu \rho \sigma \tau} A_{\mu} \partial_{v} A_{\rho} \partial_{\sigma} A_{\tau}
$$

Naturally, such a TFT describes the quantum Hall effect in the 4D space. I worked with my student $\mathrm{Hu}$ to construct a microscopic model for the 4D quantum Hall effect [12]. Even though a 4D model seems to be un-physical for condensed matter physics, we noticed a remarkable difference to the $2 \mathrm{D}$ quantum Hall effect, in that the 4D model respects time reversal symmetry while the 2D model does not! It is very easy to see the difference in the two TFTs given by equations (1) and (4). Under the time reversal transformation $\mathcal{T}$,

$$
A_{0} \rightarrow A_{0}, \quad A_{i} \rightarrow-A_{i}
$$

therefore, we see that the TFT in $2+1$ dimensions (1) breaks time-reversal symmetry, whereas the TFT in $4+1$ dimensions (4) preserves the time-reversal symmetry! 
Time reversal symmetry operation $\mathcal{T}$ in quantum mechanics come in two different classes, the orthogonal class with $\mathcal{T}^{2}=1$ and the symplectic class with $\mathcal{T}^{2}=-1$. It was easy to see explicitly that our microscopic model for the 4D quantum Hall state contains spin-orbit coupling and is in the symplectic class with $\mathcal{T}^{2}=-1$. At that moment I became convinced that there is a new topological structure in the symplectic time reversal invariant systems. In 2003, together with Murakami and Nagaosa, we discovered the intrinsic spin Hall effect in spin-orbit coupled systems [13, 14]. Since the Hall effect has a quantum version, it was then natural to ask if the spin Hall effect also has a quantum version, appropriately called the quantum spin Hall effect. Kane and Mele [15], Bernevig and I [16] independently constructed models of the quantum spin Hall effect based on spinorbit coupling in solids. At the simplest level, the quantum spin Hall state can be viewed as two copies of the quantum Hall effect, with opposite signs of the time reversal symmetry breaking, so that the combined system is invariant under time reversal symmetry. The quantum spin Hall state is insulating in the bulk, but contains gapless edge states protected by the $\mathcal{T}^{2}=-1$ symmetry.

Where in nature can one discovery new topological materials? One special material HgTe, which has a negative energy gap, came into my sight after discussions with Molenkamp. In 2006, my students and I made the connection between this material and equations (1), (2) and (3). A change of the sign of the Dirac mass implies a topological phase transition with a jump in the Chern-Simons coefficient. The topologically trivial ones have positive energy gap, and HgTe with a negative energy gap is the topological material [7]. Furthermore, HgTe has a negative energy gap because it has strong spin-orbit coupling due to the heavy element Hg. In less than a year, Molenkamp's experiment observed the helical edge states in the conductance measurement [17]. Inside the two dimensional topological insulator, the electrons move along the edges of the sample in well defined channels, very much like autos on a highway, where oppositely moving traffics are spatially separated into different lanes, thus avoiding collision with each other. Therefore, topological insulator materials could be useful for applications in electronic devices with low power dissipation.

Turning now to the 3D case, changing the sign of the Dirac mass also leads to a topological phase transition [6], inducing a topological term, called the axion electromagnetic term or the $\theta$ term [18]. We can combine the ordinary Dirac mass term $m_{4}$ with the $\mathcal{T}$ breaking mass term $m_{5}$ into a complex mass term

$$
m_{4}+i m_{5}=m e^{i \beta}
$$

Rotating the $\beta$ angle from 0 to $\pi$ adiabatically changes the sign of $m_{4}$ from positive to negative, still arriving at a $\mathcal{T}$ invariant state with $m_{5}=0$. Because of the axial anomaly in quantum field theory, such a chiral rotation induces an axion term in the electromagnetic response:

$$
\begin{aligned}
S_{\text {eff }} & =S_{\text {Maxwell }}+S_{\text {topo }} \\
& =\int d^{3} x d t\left[\frac{1}{16 \pi} F_{\mu \nu} F^{\mu v}+\frac{\theta \alpha}{32 \pi^{2}} \epsilon^{\mu \nu \sigma \tau} F_{\mu \nu} F_{\sigma \tau}\right]
\end{aligned}
$$

with $\theta=\pi$ and $\alpha=e^{2} / \hbar c$ is the fine structure constant. This effective action for 3D topological insulators can be obtained by dimensional reduction in equation (4). The $\theta$ angle changes sign under time reversal, however, the bulk theory is also periodic in $\theta$ with a period of $2 \pi$. Therefore, there are two $\mathcal{T}$ invariant massive, or gapped, states, with $\theta=0$ and $\theta=\pi$. In a $\mathcal{T}$ invariant system, $m_{5}=0$, a topological phase transition is induced by changing the sign of the Dirac mass $m_{4}$. In the language of condensed matter physics, in precise analogy with the 2D topological material HgTe, we should search for materials where a band gap inversion takes place because of the spin-orbit coupling, this has led to the discovery of 3D topological insulators materials $\mathrm{Bi}_{x} \mathrm{Sb}_{1-x}[19,20], \mathrm{Bi}_{2} \mathrm{Te}_{3}, \mathrm{Bi}_{2} \mathrm{Se}_{3}$ and $\mathrm{Sb}_{2} \mathrm{Te}_{3}[8,21,22]$.

Soon after these discovery, the study of timereversal invariant topological insulators was generalized to time-reversal invariant topological superconductors and superfluids [24, 25, 26, 27]. Topological superconductors have a full pairing gap in the bulk and gapless surface states consisting of Majorana fermions. Majorana fermions are real fermions which are their own anti-particles. There is a direct analogy between superconductors and insulators because the Bogoliubov-de Gennes (BdG) Hamiltonian for the quasiparticles of a superconductor is analogous to the Hamiltonian of a band insulator, with the superconducting gap corresponding to the band gap of the insulator. Helium three atoms are fermions, and they form a superfluid state at very low temperature. One of the superfluid state, the helium three B phase is believed to be a topological superfluid state, with a full superfluid paring gap in the bulk and gapless Majorana fermion states on the surface. Currently, many experiments are designed to observe this Majorana surface state.

Together with Qi and Witten, we obtained the topological field theory description of time-reversal invariant topological superconductors in $3+1$ dimension [28]. The topological superconductor is characterized by a topological coupling between the electromagnetic field and superconducting phase fluctuation, which has the same form as the coupling of axions with an abelian gauge field. In fact, the field 
theory description of time-reversal invariant topological superconductors in $3+1$ dimension can be regarded as a description of the boundaries of a $4+1$ dimensional topological insulator where the $U(1)$ gauge symmetry on the boundaries is broken,

$$
S_{\mathrm{CS}}=\frac{1}{32 \pi^{2}} \int d^{4} x \epsilon^{\mu \nu \sigma \tau} \frac{\theta_{L}-\theta_{R}}{2} F_{\mu \nu} F_{\sigma \tau},
$$

where $F_{\mu \nu}=\partial_{\mu} A_{v}-\partial_{v} A_{\mu}$ is the $3+1$ dimensional electromagnetic field. $\theta_{L}$ and $\theta_{R}$ are the $U(1)$ superconducting phase field on left and right boundaries, respectively. Clearly, the $U(1)$ field $\theta_{L}-\theta_{R}$ is coupled to the gauge field as an axion field. Together the Maxwell term and the free energy that leads to the topological superconductor phase, the full electromagnetic response is

$$
\begin{aligned}
S_{\text {eff }}= & S_{\text {Maxwell }}+S_{\text {topo }}+S_{\mathrm{SC}} \\
= & \int d^{4} x\left[-\frac{1}{4} F_{\mu \nu} F^{\mu \nu}+\frac{\theta_{L}-\theta_{R}}{64 \pi^{2}} \epsilon^{\mu v \sigma \tau} F_{\mu \nu} F_{\sigma \tau}\right. \\
& +\frac{1}{2} \rho_{L}\left(\partial_{\mu} \theta_{L}-2 A_{\mu}\right)^{2}+\frac{1}{2} \rho_{R}\left(\partial_{\mu} \theta_{R}-2 A_{\mu}\right)^{2} \\
& \left.+J \cos \left(\theta_{L}-\theta_{R}\right)\right] .
\end{aligned}
$$

In the absence of gauge fields, this action gives the topological superconductor phase $\theta_{L}=0, \theta_{R}=\pi$. In the $3+1$ dimension topological superconductor, $\theta_{L}$ and $\theta_{R}$ represents the superconducting phase of two Fermi surfaces which are time reversal of each other. This approach can be generalized to topological superconductor with topological invariant $\mathcal{N}$ by considering a $4+1$ dimensional topological insulator with second Chern number $C_{2}=\mathcal{N}$.

Topology started out as an abstract branch of mathematics, but now finds many applications in condensed matter physics, with testable experimental predictions. Mathematician developed ChernSimons term, Pontryagin index, Chern numbers and index theorems to classify abstract mathematical shapes. These deep concepts have all found natural counterparts in topological insulators and superconductors. Remarkable, these ideas have directly led to the theoretical prediction of actual material systems which were later observed experimentally. On the occasion of the establishment of the Harvard center for mathematical science, I would like to wish great success of the center. May the center not only promote the the development of pure and abstract mathemat- ics, but also become a platform for cross fertilization with many other branches of science.

\section{Acknowledgments}

This work was supported by the NSF under grant numbers DMR-1305677.

\section{References}

[1] X. L. Qi and S. C. Zhang, Phys. Today 63, 33 (2010)

[2] X. L. Qi and S. C. Zhang, Rev. Mod. Phys. 83, 1057 (2011)

[3] M. Z. Hasan and C. L. Kane, Rev. Mod. Phys. 82, 3045 (2010)

[4] J. Moore, Nature 464, 194 (2010)

[5] S. C. Zhang, Int. J. Mod. Phys. B 6, 25 (1992)

[6] X. L. Qi, T. Hughes, and S. C. Zhang, Phys. Rev. B 78, $195424(2008)$

[7] B. A. Bernevig, T. L. Hughes, and S. C. Zhang, Science 314, 1757 (2006)

[8] H. Zhang, C.-X. Liu, X.-L. Qi, X. Dai, Z. Fang, and S.-C. Zhang, Nature Phys. 5, 438 (2009)

[9] R. J. S. Deser and S. Templeton, Phys. Rev. Lett. 48, 975 (1982)

[10] A. J. Niemi and G. W. Semenoff, Phys. Rev. Lett. 51, 2077 (1983)

[11] A. N. Redlich, Phys. Rev. D 29, 2366 (1984)

[12] S. C. Zhang and J. P. Hu, Science 294, 823 (2001)

[13] S. Murakami, N. Nagaosa, and S. C. Zhang, Science 301, 1348 (2003)

[14] J. Sinova, D. Culcer, Q. Niu, N. A. Sinitsyn, T. Jungwirth, and A. H. MacDonald, Phys. Rev. Lett. 92, 126603 (2004)

[15] C. L. Kane and E. J. Mele, Phys. Rev. Lett. 95, 226801 (2005)

[16] B. A. Bernevig and S. C. Zhang, Phys. Rev. Lett. 96, 106802 (2006)

[17] M. König, S. Wiedmann, C. Brüne, A. Roth, H. Buhmann, L. Molenkamp, X.-L. Qi, and S.-C. Zhang, Science 318, 766 (2007)

[18] F. Wilczek, Phys. Rev. Lett. 58, 1799 (1987)

[19] L. Fu and C. L. Kane, Phys. Rev. B 76, 045302 (2007)

[20] D. Hsieh, D. Qian, L. Wray, Y. Xia, Y. S. Hor, R. J. Cava, and M. Z. Hasan, Nature 452, 970 (2008)

[21] Y. Xia, L. Wray, D. Qian, D. Hsieh, A. Pal, H. Lin, A. Bansil, D. Grauer, Y. Hor, R. Cava, and M. Hasan, Nature Phys. 5, 398 (2009)

[22] Y. L. Chen, J. G. Analytis, J. H. Chu, Z. K. Liu, S.-K. Mo, X. L. Qi, H. J. Zhang, D. H. Lu, X. Dai, Z. Fang, S. C. Zhang, I. R. Fisher, Z. Hussain, and Z.-X. Shen, Science 325, 178 (2009)

[23] R. Roy, Phys. Rev. B 79, 195321 (2009)

[24] R. Roy, arXiv:0803.2868 (2008)

[25] A. P. Schnyder, S. Ryu, A. Furusaki, and A. W. W. Ludwig, Phys. Rev. B 78, 195125 (2008)

[26] A. Kitaev, AIP Conf. Proc. 1134, 22 (2009)

[27] X.-L. Qi, T. L. Hughes, S. Raghu, and S.-C. Zhang, Phys. Rev. Lett. 102, 187001 (2009)

[28] X.-L. Qi, E. Witten, and S.-C. Zhang, Phys. Rev. B 87, $134519(2013)$ 